\title{
High-performance liquid chromatography for the determination of oxymatrine content in radix Sophorae tonkinensis
}

\author{
Changli Shao MSc${ }^{1}$, Chuling Du MSc${ }^{1}$, Jingsheng Du MSc${ }^{2}, X_{i}$ Xia MSc ${ }^{1}$, \\ Yinghong Huang $\mathrm{MSc}^{1}$, Yu Cai $\mathrm{PhD}^{1}$, Zhihai Huang $\mathrm{MSc}^{2}$
}

\begin{abstract}
C Shao, C Du, J Du, et al. High-performance liquid chromatography for the determination of oxymatrine content in radix Sophorae tonkinensis. Curr Res Integr Med 2015;1(1):8-10.
\end{abstract}

The purpose of the present study was to establish a high-performance liquid chromatography method for the determination of the oxymatrine content of radix Sophorae tonkinensis and to comparatively analyze the content of oxymatrine in radix Sophorae tonkinensis from different origins. C18 was used as a chromatographic column $(250 \mathrm{~mm} \times 4.6 \mathrm{~mm}, 5 \mu \mathrm{m})$, metha$\mathrm{nol} / 3 \%$ phosphoric acid in water $(80: 20)$ as the mobile phase, $220 \mathrm{~nm}$ as the ultraviolet wavelength detection, $1 \mathrm{~mL} / \mathrm{min}$ as the flow rate and $30^{\circ} \mathrm{C}$ as the column temperature. All samples were subjected to organic solvent

$\mathbf{P}$ adix Sophorae tonkinensis is the processed lateral root of Sophorae Rtonkinensis Gapnep (1). It is bitter, cold, toxic, in the lung and stomach meridian. It has been used as a Chinese medicinal herb to treat jaundice, inflammation and aches, and to clear away heat and purging pathogenic fire (2,3). Radix Sophorae tonkinensis mainly include alkaloid, flavonoids and triterpenoid, but oxymatrine and matrine are the primary effective components $(4,5)$. However, oxymatrine and matrine are also the primary toxic components. The Pharmacopoea of the People's Republic of China states that the safe and effective dosage of radix Sophorae tonkinensis is $3 \mathrm{~g}$ to $6 \mathrm{~g}$. If the dosage exceeds $12 \mathrm{~g}$, it can cause severe adverse reactions such as nausea and vomiting (6,7). Radix Sophorae tonkinensis from different origins have different oxymatrine content. The aim of the present study was to establish a highperformance liquid chromatography (HPLC) method for the determination of oxymatrine, and to determine the oxymatrine content of different origins of radix Sophorae tonkinensis to provide an evaluation standard for controlling radix Sophorae tonkinensis quality.

\section{METHODS}

\section{Materials}

The reference substance of oxymatrine was purchased from Guangzhou Institute for Drug Control (Guangzhou, China). Radix Sophorae tonkinensis was provided by The Second Affiliated Hospital of Guangzhou University of Traditional Chinese Medicine (Guangzhou, China). Methanol and phosphoric acid were chromatographic grade, and all the other chemicals were analytical grade.

\section{Instrumentation}

All HPLC experiments were performed on a self-assembled HPLC (SHIMADZU, Japan), with the C18 chromatographic column (R\&C, Guangzhou). The extraction process of radix Sophorae tonkinensis used rotary evaporators (EYELA, Shanghai), ultrasonic cleaner (Ningbo, China) and an electronic balance (ACCULAB, Beijing). extraction with ultrasonic treatment before the oxymatrine content from radix Sophorae tonkinensis was measured. Oxymatrine in the range of 30 $\mu \mathrm{g} / \mathrm{mL}$ to $150 \mu \mathrm{g} / \mathrm{mL}$ had a good linear relationship ( $\mathrm{r}=0.9998)$, and the average recovery was $99.22 \%$, suggesting that this method is accurate and has high reproducibility. The established high-performance liquid chromatography method for determining oxymatrine content from radix Sophorae tonkinensis is efficient and stable. This may be an evaluation criteria used for quality control given that different origins of radix Sophorae tonkinensis have wide-ranging differences in oxymatrine content.

Key Words: HPLC; Oxymatrine; radix Sophorae tonkinensis

Chromatographic conditions

C18 as chromatographic column $(250 \mathrm{~mm} \times 4.6 \mathrm{~mm}, 5 \mu \mathrm{m})$, methanol/3\% phosphoric acid in water $(80: 20)$ as the mobile phase, $220 \mathrm{~nm}$ as the ultraviolet wavelength detection, $1 \mathrm{~mL} / \mathrm{min}$ as the flow rate, $30^{\circ} \mathrm{C}$ as the column temperature.

\section{Oxymatrine standard preparation}

$7.5 \mathrm{mg}$ oxymatrine standard was precisely measured and dissolved in methanol in a $50 \mathrm{~mL}$ volumetric flask, obtaining a $150 \mu \mathrm{g} / \mathrm{mL}$ oxymatrine standard solution. Subsequently, $2 \mathrm{~mL}, 4 \mathrm{~mL}, 6 \mathrm{~mL}$ and $8 \mathrm{~mL}$ of the solution was diluted with methanol to obtain $30 \mu \mathrm{g} / \mathrm{mL}, 60 \mu \mathrm{g} / \mathrm{mL}$, $90 \mu \mathrm{g} / \mathrm{mL}$ and $120 \mu \mathrm{g} / \mathrm{mL}$ oxymatrine standard solutions.

\section{Preparing radix Sophorae tonkinensis samples}

Radix Sophorae tonkinensis was crushed and screened, then taking screened powder (approximately $0.5 \mathrm{~g}$ ) gave a respective weight, denoted M. Trichloromethane-methanol-ammonia (40:10:1) was used to dispose the radix Sophorae tonkinensis samples for $30 \mathrm{~min}$, and all samples were subsequently obtained from organic solvent extraction with $30 \mathrm{~min}$ ultrasonic treatment. All disposed samples were filtered, then $10 \mathrm{~mL}$ of filtrate was measured to recover solvents to dry under decompression at $38^{\circ} \mathrm{C}$ to obtain the residue, the residue was diluted by methanol, then transferred to a $10 \mathrm{~mL}$ volumetric flask. After mixing and filtering with $0.45 \mu \mathrm{m}$ filter membrane, radix Sophorae samples were obtained. The blank groups were treated as the samples but without radix Sophorae tonkinensis.

\section{The content of oxymatrine}

Twenty microlitres of radix Sophorae tonkinensis samples were injected in the HPLC system, the peak area was recorded, mass concentration (C) was obtained and the content of Oxymatrine from radix Sophorae tonkinensis was determined. The content of Oxymatrine was calculated by $51 \mathrm{C} / 1000 \mathrm{M}$.

\footnotetext{
${ }^{1}$ College of Pharmacy, JiNan University; ${ }^{2}$ Second Affiliated Hospital of Guangzhou University of Chinese Medicine, Guangzhou, China

Correspondence: Yu Cai, 601 Huangpu Street West, Tianhe District, Guangzhou, China 510632. Telephone 86-13119582329, e-mail 1710001232@qq.com; Zhihai Huang, 55 Huanxi Road, University City, Panyu District, Guangzhou, China 510006.

Telephone 86-02-81887233,e-mail 1-long-1@163.com
} 


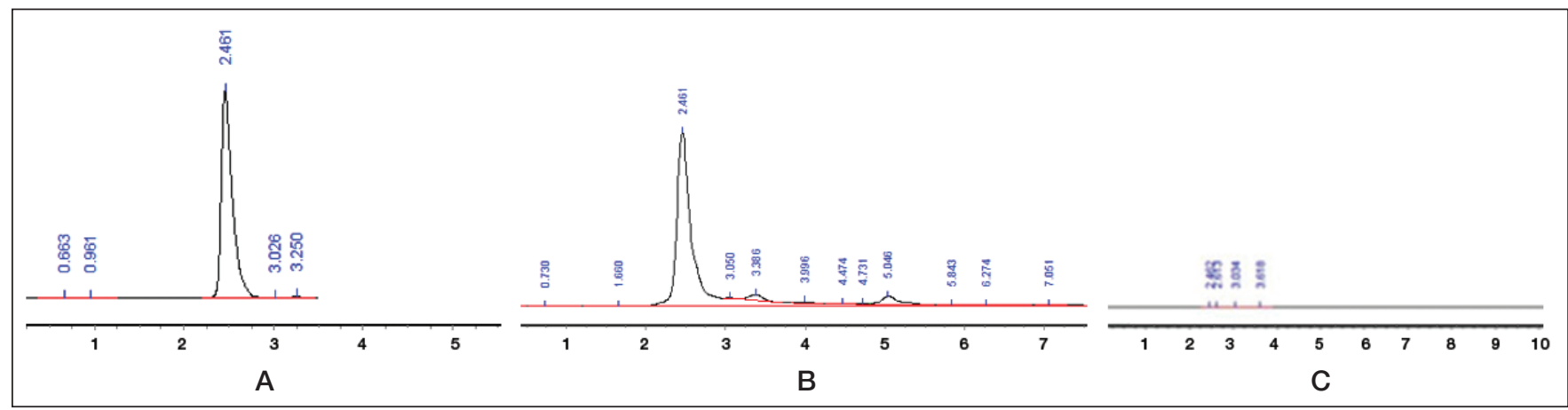

Figure 1) High-performance liquid chromotography chromatograms of oxymatrine. A Standard, B sample, C Blank

TABLE 1

Results of recovery tests $(n=3)$

\begin{tabular}{lccccc}
\hline NO* & Samples contained, $\mathbf{m g}$ & Added, $\mathbf{m g}$ & Measured, $\mathbf{m g}$ & Recovery, \% & Average recovery, \% Relative SD, \% \\
\hline 1 & 5.707 & 0.150 & 5.854 & 98.00 & \\
2 & 5.710 & 0.150 & 5.859 & 99.33 & \\
3 & 5.703 & 0.150 & 5.852 & 99.33 & \\
4 & 5.703 & 0.150 & 5.849 & 97.33 & 102.67 \\
5 & 5.700 & 0.150 & 5.854 & 98.67 & 99.22 \\
6 & 5.705 & 0.150 & 5.853 & & 1.874 \\
Total & & & & & \\
\hline
\end{tabular}

*All Radix Sophorae tonkinensis were from Guangxi, China, batch number: 121003; the content of oxymatrine was $22.82 \mathrm{mg} / \mathrm{g}$

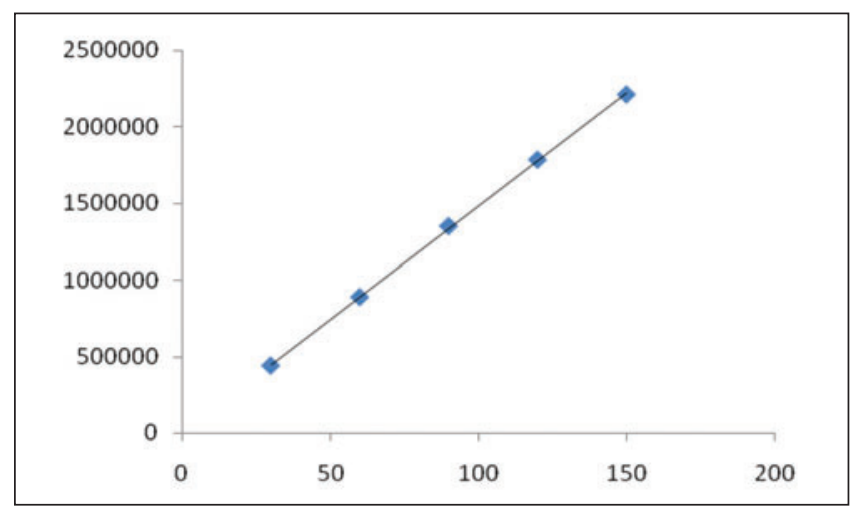

Figure 2) Standard curve of oxymatrine

Specific inspection study

\section{RESULTS}

According to the described chromatographic conditions, oxymatrine standard and radix Sophorae tonkinensis samples were effective separated. The other ingredients did not interfere with the oxymatrine chromatography peak. The method had high specificity; specificity results are shown in Figure 1.

Standard curve drawing

With peak area $(y)$ as the ordinate and mass concentration $(\mathrm{x}, \mu \mathrm{g} / \mathrm{mL})$ as the abscissa, the standard curves for oxymatrine were linear in the concentration range of $30 \mu \mathrm{g} / \mathrm{mL}$ to $150 \mu \mathrm{g} / \mathrm{mL}$ ( $y=14739 \mathrm{x}+9487.6$, $\mathrm{r}=0.9998)$; a standard curve is shown in Figure 2.

Precision, stability, repeatability and recovery tests

Taking the same concentration of oxymatrine standard solution, continuously investigated six times, the RSD result of precision test was $0.941 \%$; taking screened radix Sophorae tonkinensis powder (approximately $0.5 \mathrm{~g}$ ), disposing samples as described, then investigating its peak area every $2 \mathrm{~h}$, the RSD result of stability test was $1.423 \%$ in $12 \mathrm{~h}$; taking screened radix Sophorae tonkinensis powder
TABLE 2

Results of different origin of Radix Sophorae tonkinensis sample determination $(n=3)$

\begin{tabular}{lcc}
\hline Origin & Batch number & Oxymatrine content, $\mathbf{~ g / g}$ \\
\hline Heilongjiang, China & 121001 & 24.72 \\
& 121002 & 24.85 \\
Guilin, Guangxi, China & 121003 & 22.82 \\
& 1209021411 & 7.18 \\
& 1209021412 & 6.98 \\
Nanning, Guangxi, China & 1209021413 & 6.99 \\
& 120901 & 9.28 \\
& 120902 & 8.43 \\
& 120903 & 8.27 \\
\hline
\end{tabular}

(approximately $0.5 \mathrm{~g}$ ), disposing samples as described, then continuously investigated its peak area six times, the RSD result of repeatability test was $1.879 \%$; taking screened radix Sophorae tonkinensis powder (approximately $0.25 \mathrm{~g}$ ) for the six groups, disposing them as described, then adding $0.150 \mathrm{mg}$ oxymatrine standard solution to the six radix Sophorae tonkinensis samples and investigating its peak area, the average recovery was $99.22 \%$, and RSD was $1.874 \%$. The results of recovery tests are shown in Table 1. Precision, stability, repeatability and recovery tests were well within the $5 \%$ acceptable range.

Oxymatrine content of different origin radix Sophorae tonkinensis samples

Approximately $0.5 \mathrm{~g}$ of radix Sophorae tonkinensis powder from different origins disposed them as described, recording the peak area, the content of oxymatrine from radix Sophorae tonkinensis was obtained and is presented in Table 2 .

As the results in Table 2 demonstrate, radix Sophorae tonkinensis coming from Heilongjiang origin had the highest oxymatrine content 
$24.85 \mathrm{mg} / \mathrm{g}$. Radix Sophorae tonkinensis of Guilin origin had the lowest content, and the highest content was approximately four times the lowest (a considerable difference).

\section{DISCUSSION}

Oxymatrine has a unique oxygen structure; it can lower blood pressure, resist viruses, strengthen the heart, resist arrhythmia, and treat jaundice, inflammation and aches. Existing research shows that oxymatrine has antitumour activity, mainly by inhibiting DNA synthesis in tumour cells and restraining enzyme activity to inhibit the growth of tumour cells (8). Oxymatrine is the one of the main effective ingredients of radix Sophorae tonkinensis; studying the different oxymatrine content from different origins of radix Sophorae tonkinensis, the peak concentration was $24.85 \mathrm{mg} / \mathrm{g}$, but the lowest concentration was $7.18 \mathrm{mg} / \mathrm{g}$. The reasons are as follows: different geographical environment causes the different oxymatrine content, and different growth periods lead to the difference $(9,10)$.

Choosing amino as chromatographic column, acetonitrile/isopropyl alcohol $/ 3 \%$ phosphoric acid in water $(80: 5: 15)$ as the mobile phase,

\section{REFERENCES}

1. Wang P, Wu X, Li JP, et al. Acute toxicity experiment of Radix Sophorae tonkinensis. Lishizhen Medicine and Materia Medica Research 2013;04:771-3.

2. Kang ZY, Yu CP. The extraction process of Radix Sophorae tonkinensis research and discussion. Zhongyaocai 2007;1:101-2.

3. China Pharmacopoeia Committee. Chinese pharmacopoeia. Chinese medical science and technology publishing 2010;1:25-6.

4. H Yafei, $\mathrm{H}$ Jiwei, T Ling, et al. Studies on fingerprint spectrum of Radix Sophorae Tonkinensis from Guangxi. Zhongyaocai 2005;281-3.

5. Ding PL, Chen DF. phenolic compounds research of Radix Sophorae tonkinensis. Zhongcaoyao 2008;2:186-8.

6. Song MQ, Zhu JS, Chen JL, et al. Synergistic effect of oxymatrine and angiogenesis inhibitor NM-3 on modulating apoptosis in human gastric cancer cells. World J Gastroenterol 2007;13:1788-93. chromatographic peak of oxymatrine appeared overlapping; choosing C18 as chromatographic column, methanol/2.5\% phosphoric acid in water $(5: 95)$ as the mobile phase, the chromatography of oxymatrine appeared baseline drift; choosing C18 as chromatographic column, methanol/water (75:25) as the mobile phase, the chromatography of oxymatrine could not be separated. Finally, choosing C18 as chromatographic column $(250 \times 4.6 \mathrm{~mm}, 5 \mu \mathrm{m})$, methanol $/ 3 \%$ phosphoric acid in water $(80: 20)$ as the mobile phase, $220 \mathrm{~nm}$ as the ultraviolet wavelength detection, $1 \mathrm{~mL} / \mathrm{min}$ as the flow rate and $30^{\circ} \mathrm{C}$ as the column temperature, good specificity was obtained.

The present study established a HPLC methodology for determination of oxymatrine content from radix Sophorae tonkinensis. Because oxymatrine is one of the main effective ingredients and is stable, the content of oxymatrine can be a standard for controlling and evaluating the quality of radix Sophorae tonkinensis to offer theoretical basis.

FOUNDATION SUPPORT: Science and Technology Program of Guangdong, China; Project number: 2012B031800200.

7. Y Long, XT Lin, KL Zeng, et al. Efficacy of intramuscular matrine in the treatment of chronic hepatitis B. Hepatobiliary Pancreat Dis Int 2004;3:69-72.

8. Hou Y, Cao W, Li T, et al. Oxymatrine induced HepG2 apoptosis of liver cancer possible mechanism. Academic Journal of Second Military Medical University 2008;29:634-8.

9. Peng YH, Jiang CY, Lin W, et al. Matrine and oxymatrine content in different growth duration of Radix Sophorae tonkinensis. Chinese Journal of Experimental Traditional Medical Formulae 2014;8:72-5.

10. Huang YF, Huang JW, Tao L, et al. Studies on fingerprint spectrum of Radix Sophorae Tonkinensis from Guangxi. Zhongyaocai 2005;281-3. 